\title{
Fitting of Radial Dose Function for 192ir Brachytherapy Sources
}

\author{
AV Belousov*1, UA Bliznyuk² ${ }^{2}$ AA Belyanov² and GA Krusanov ${ }^{1}$ \\ ${ }^{1}$ Physics Department, MV. Lomonosov Moscow State University, Moscow, Russia \\ ${ }^{2}$ Nuclear research institute named by DV. Skobeltcin, Moscow, Russia
}

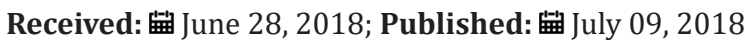

*Corresponding author: AV Belousov,Physics Department, MV Lomonosov Moscow State University, Moscow, Russia

\begin{abstract}
This article presents an improved configuration for fitting the radial dose functions of sealed 192Ir brachytherapy sources. The proposed functions allow for precise fitting of radial dose function in the range of $0.520 \mathrm{~cm}$. The maximum deviations between fitting data and Monte Carlo modeling data do not exceed $0.6 \%$, while average deviations are in the order of $0.1 \%$. The fitting coefficients for the proposed functions are specified for 11 sources from different manufacturers. The proposed functions could be used in treatment planning systems for more accurate calculation of the dose distributions around the sources using a wide range of data according to TG-43 dosimetry protocol.
\end{abstract}

Keywords: Brachytherapy; Monte Carlo; Fitting Radial Dose Function

Abbrevations: AAPM: American Association of Physicists in Medicine; TG-43: Task Group 43

\section{Introduction}

In order to calculate the absorbed dose distribution near the brachytherapy source many commercial treatment planning systems use functional dependencies obtained by fitting a set of values of the radial dose function. This dataset could be determined by experimental dosimetry studies or by computer simulation. The American Association of Physicists in Medicine (AAPM) Task Group 43 (TG-43) dosimetry protocol recommends using third to fifth order polynomials in the following form [1].

$$
g(r)=a_{0}+a_{1} r+a_{2} r^{2}+a_{3} r^{3}+a_{4} r^{4}+a_{5} r^{5} .
$$

The fit coefficients are available in scientific publications for a large number of currently used sources. Although the accuracy of the fifth order polynomials is sufficient for fitting and interpolation of the radial dose function within the range $0.5 \mathrm{~cm} \leq r \leq 20 \mathrm{~cm}$ he fitting quality decreases sharply beyond this range. Also, the behavior of the function is 'non-physical' outside this range. This is manifested by the large number of minima and maxima. Various authors have proposed many alternatives to polynomials, including a double exponential fit [2]:

$$
g(r)=a_{0} e^{-a_{1} r}+a_{2} e^{-a_{3} r},
$$

Moss [3] proposed using a modified sigmoid function:

$$
g(r)=a_{0}\left(\frac{a_{1}+e^{-a_{2}\left(r-a_{4}\right)}}{a_{1}+e^{-a_{2}\left(r-a_{4}\right)}+e^{-a_{3}\left(r-a_{4}\right)}}\right),
$$

Meigooni [4] proposed modifying the polynomial by an exponential factor:

$$
g(r)=\left(a_{0}+a_{1} r+a_{2} r^{2}+a_{3} r^{3}+a_{4} r^{4}+a_{5} r^{5}\right) e^{-a_{6} r} .
$$

Even though all of the above functions allow for a more accurate fitting of data, yet they don't always accurately describe the behavior of the radial dose function at small distances from the source, which is especially crucial for sources with ${ }^{125} \mathrm{I}$ and ${ }^{103} \mathrm{Pd}$ radionuclides. Expression (2) has some physical sense if we assume that the spectrum of the photons emitted by the radionuclide, consists of two main lines, which is true for ${ }^{60} \mathrm{Co}$. In order to better reflect the rapid change of the radial dose function at small distances from the source, expression (4) could be modified as follows:

$$
g(r)=\left(a_{0} r^{-1}+a_{1}+a_{2} r+a_{3} r^{2}+a_{4} r^{3}\right) e^{-a_{6} r} \equiv P_{-1,3} \times \exp ^{\prime}
$$

or in the form [5]:

$$
g(r)=\left(a_{0} r^{-2}+a_{1} r^{-1}+a_{2}+a_{3} r+a_{4} r^{2}+a_{5} r^{3}\right) e^{-a_{6} r} \equiv P_{-2} \times \exp
$$

or

$$
g(r)=\left(a_{0} r^{-1}+a_{1}+a_{2} r+a_{3} r^{2}+a_{4} r^{3}+a_{5} r^{4}\right) e^{-a_{6} r} \equiv P_{-1,4} \times \exp
$$

All expressions are compiled in such a way that the number of fitting parameters $\mathrm{a}_{0}, \mathrm{a}_{1}, .$. , would not exceed 7. In this research, we calculated the radial dose function for source BEBIGIr2.A95-2, 
performed fitting by formulas (1) and (4-6) for this source and 10 other sources with ${ }^{192}$ Ir used as a radionuclide, as well as performed fitting by a sixth order polynomial and Gaussian function.

\section{Materials and Methods}

Two types of brachytherapy sources containing ${ }^{192} \mathrm{Ir}$ as an active substance are commercially available. The first type is characterized by a gradually increasing radial dose function which reaches its maximum at the distance of $1 \mathrm{~cm}$ from the source; whereas the second type is characterized by a dose decrease with distance at small values of $r$. Among the type one sources are BEBIG GI192M11, Flexisource, Gammamed 12i, Gammamed plus, Microselectron V1 and V2, SPEC M19, Vari Source classic and Vary Source VS200; the type two sources are BEBIG Ir2.A95-2 and Buchler G0814. Values of the radial dose functions for source BEBIG Ir2.A95-2 were obtained by the authors [6], and taken from publication [7]; data for all other sources is taken from the database of Carleton University [8]. In all cases, the nonlinear least-squares fitting was performed using OriginPro software. The fitting quality was assessed by two criteria: by the error of fit coefficients and by the average and maximum deviations of the fitting results from the tabulated data, according to the following expressions:

$$
R_{i}=\left|\frac{g_{\text {fit }}\left(r_{i}\right)-g_{\text {data }}\left(r_{i}\right)}{g_{\text {data }}\left(r_{i}\right)}\right|,
$$

$$
\langle R\rangle=\frac{1}{N} \sqrt{\sum_{i=1}^{i=N} R_{i}^{2}}
$$

where $g_{f i t}\left(r_{i}\right)$ is the result of the fitting function calculation at point $r_{i}, g_{\text {data }}\left(r_{i}\right)$ is the value of tabulated Monte Carlo data at the same point. The fitting of each function was performed in the range $0.5 \mathrm{~cm} \leq r \leq 20 \mathrm{~cm}$.

\section{Results and Discussion}

The best fitting results for the radial dose functions of the type one sources is provided by formulas (5) through (7). For the type two sources, the best quality fitting is obtained by formulas (6) and (7)(Table 1). Overall, fitting by formula (7) provides the most satisfactory results for all sources studied, because at equal quantity of parameters provides a smaller average derivations fitting data and a smaller error in parameter definitions. Fitting parameters for formulas (5) - up and (7) - down, are presented in Table 1 . The maximum deviations between fitting data and Monte Carlo modeling data do not exceed $0.4 \%$ for all source, whereas average deviations are in the order of 0.1 sssssss $\%$. The proposed functions could be used in treatment planning systems for more accurate calculation of the dose distributions around the sources using a wide range of data according to TG-43 dosimetry protocol.

\begin{tabular}{|c|c|c|c|c|c|c|c|c|c|}
\hline Source & $a_{o} \times 10^{-3} \mathbf{c m}$ & $a_{1} \times 10^{-1} \mathrm{~cm}$ & $a_{2} \times 10^{-2} \mathbf{c m}^{-1}$ & $a_{3} \times 10^{-3} \mathbf{c m}^{-2}$ & $a_{4} \times 10^{-4} \mathbf{c m}^{-3}$ & $a_{5} \times 10^{-7} \mathbf{c m}^{-4}$ & $a_{6} \times 10^{-1}, \mathbf{c m}^{-1}$ & $<\mathrm{R}>, \%$ & $\mathbf{R}_{\max }, \%$ \\
\hline \multirow{2}{*}{ BEBIG GI192M11 } & -4.03 & 9.96 & 12.4 & 7.04 & 1.26 & 0 & 1.18 & 0.07 & 0.22 \\
\hline & 4.08 & 9.97 & 97.2 & 4.19 & -0.31 & -4.18 & 0.92 & 0.07 & 0.22 \\
\hline \multirow{2}{*}{ FlexiSource } & -1.98 & & 10.92 & 5.53 & 2.29 & 0 & 1.03 & 0.07 & 0.25 \\
\hline & -2.38 & 9.95 & 5.62 & 1.16 & -1.39 & 26 & 0.51 & 0.07 & 0.2 \\
\hline \multirow{2}{*}{ Gammamed $12 \mathrm{i}$} & -5.19 & 9.97 & 12.08 & 6.89 & 0.94 & 0 & 1.16 & 0.1 & 0.33 \\
\hline & -5.29 & 9.98 & 13.53 & 9.03 & 2.05 & 33.41 & 1.32 & 0.1 & 0.33 \\
\hline \multirow{2}{*}{ Gammamed plus } & -3.79 & 9.96 & 12.14 & 6.82 & 0.95 & 0 & 1.16 & 0.07 & 0.26 \\
\hline & -3.79 & 9.96 & 12.16 & 6.84 & 0.96 & 0.17 & 1.16 & 0.06 & 0.26 \\
\hline \multirow{2}{*}{ Microselectron V1 } & -1.97 & 9.92 & 13.12 & 7.39 & 0.2 & 0 & 1.24 & 0.09 & 0.38 \\
\hline & -1.97 & 9.92 & 13.03 & 7.35 & 0.2 & -0.64 & 1.24 & 0.09 & 0.38 \\
\hline \multirow{2}{*}{ Microselectron V2 } & -2.28 & 9.85 & 13.22 & 6.29 & 0.16 & 0 & 1.19 & 0.07 & 0.21 \\
\hline & 0.66 & 9.9 & 16.34 & 12.91 & 0.29 & 203 & 1.54 & 0.07 & 0.16 \\
\hline \multirow{2}{*}{ SPEC M19 } & -2.62 & 9.95 & 13.02 & 7.29 & 0.18 & 0 & 1.23 & 0.1 & 0.27 \\
\hline & -2.62 & 9.95 & 13.06 & 7.28 & 0.18 & -0.1 & 1.23 & 0.1 & 0.27 \\
\hline \multirow{2}{*}{ VariSource Classic } & -7.1 & 9.98 & 11.12 & 5.39 & 3.07 & 0 & 1.02 & 0.08 & 0.19 \\
\hline & -9.75 & 10 & 14.07 & 11.07 & 1.27 & 86.6 & 1.37 & 0.08 & 0.18 \\
\hline \multirow{2}{*}{ VariSource V2000 } & 1.34 & 9.87 & 13.11 & $6.44 \mathrm{E}-03$ & 1.39 & 0 & 1.18 & 0.08 & 0.21 \\
\hline & 1.35 & 9.87 & 13.23 & $6.57 \mathrm{E}-03$ & 1.46 & 1.29 & 1.19 & 0.07 & 0.21 \\
\hline \multirow{2}{*}{ Buchler G0814 } & 10.1 & 9.78 & 15.09 & 7.24 & 3.64 & 0 & 1.32 & 0.08 & 0.22 \\
\hline & 10.1 & 9.78 & 15.07 & 7.24 & 3.64 & 0 & 1.32 & 0.08 & 0.21 \\
\hline \multirow{2}{*}{ BEBIG Ir2.A95-2 [6] } & 20.4 & 9.91 & 15.56 & -0.48 & 0 & 0 & 0.16 & 0.09 & 0.22 \\
\hline & 19.3 & 9.98 & 13.22 & 10.51 & 0.9 & 260 & 1.48 & 0.08 & 0.22 \\
\hline \multirow{2}{*}{ BEBIG Ir2.A95-2 [7] } & 32.5 & 9.72 & 11.31 & 1.93 & 2.45 & 0 & 1.1 & 0.09 & 0.25 \\
\hline & 36.7 & 9.61 & 14.85 & 2.54 & 6.08 & -0.21 & 1.36 & 0.09 & 0.23 \\
\hline
\end{tabular}

Table 1: Fitting Parameters. 


\section{Conclusion}

In this article, we propose fitting functions for the radial dose function of sealed high-dose ${ }^{192}$ Ir brachytherapy sources. The proposed functions were used for fitting the data for 11 sources obtained by the authors using the Monte Carlo method as well as data available from the database of Carleton University. The proposed functions result in higher quality fitting compared to fifth order polynomial fits recommended by AAPM TG-43. These functions can be used at distances $0.5-20 \mathrm{~cm}$ from the source. The maximum deviations between fitting data and Monte Carlo modeling data do not exceed $0.6 \%$, whereas average deviations are in the order of $0.1 \%$. We believe that the proposed functions are excellent candidates for use in calculating dose distributions in treatment planning systems.

\section{References}

1. Nath R, Anderson LL, G Luxton, Weaver KA, Williamson JF, et al. (1995) Dosimetry of interstitial brachytherapy sources: Recommendations of the AAPM Radiation Therapy Committee Task Group No. 43. Medical Physics 22(2): 209-234.

\section{ISSN: 2574-1241}

DOI: 10.26717/BJSTR.2018.06.001370

AV Belousov. Biomed J Sci \& Tech Res

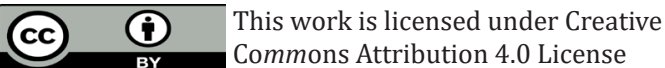

Submission Link: https://biomedres.us/submit-manuscript.php
2. Furhang EE, Anderson LL (1999) Functional fitting of interstitial brachytherapy dosimetry data recommended bu the AAPM Radiation Therapy Committee Task Group, No. 43. Medical Physics 26(2): 153-160.

3. Moss D (2000) Technical note: Improved analytical fit to the TG-43 radial dose function. Medical Physics 27(4): 659-661.

4. Meigooni AS, Zhang H, Perry C, Dini SA, Koona RA (2003) Theoretical and experimental determination of dosimetric characteristics for brachyseed Pd-103 model Pd-1 source. Applied radiation and isotopes 58(5): 533-554.

5. Taylor REP, Rogers DWO (2008) More accurate fitting of $125 \mathrm{I}$ and $103 \mathrm{Pd}$ radial dose functions. Medical Physics 35(9): 4242-4250.

6. Belousov AV, Kalachev AA, Osipov AS (2014) Monte Carlo Calculation of Dosimetry Parameters for Brachytherapy Sources. Moscow University Bulletin. Series 3. Physics. Astronomy, No. 69(6): 535-541.

7. Granero D, Perez-Calatayud J, Ballester F (2008) Monte Carlo study of the dose rate distributions for the IR.A85-2 and IR.A85-1 Ir-192 afterloading sources. Medical Physics 35(4): 1280-1287.

8. REP Taylor, DWO Rogers (2013) The CLRP TG-43 Parameter Database for Brachytherapy. Carleton Laboratory for Radiotherapy Physics.

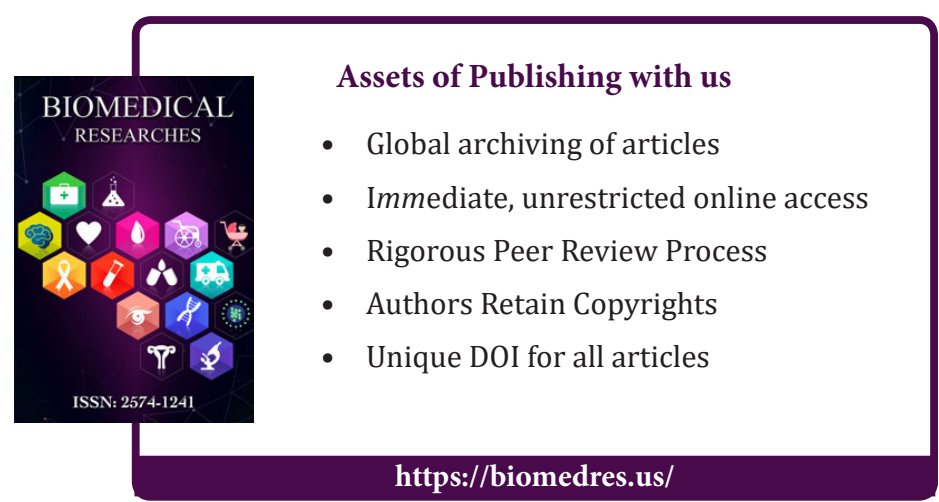

